\title{
Strategic Behavior, Private Information, and Decentralization in the European Union Emissions Trading System
}

\author{
David A. Malueg • Andrew J. Yates
}

Accepted: 10 February 2009 / Published online: 3 March 2009

(C) The Author(s) 2009. This article is published with open access at Springerlink.com

\begin{abstract}
In the European Union Emissions Trading System, the supply of carbon permits is determined in a decentralized manner by Member States. Alternatively, the supply could be determined by an EU central authority. We analyze whether decentralization leads to lower total abatement costs under various assumptions about the behavior of Member States and the privacy of information about their abatement costs. If Member States do not behave strategically, then decentralization is preferred, regardless of whether abatement costs are private information. If the Member States behave strategically, then decentralization may or may not be preferred, depending on the degree of uncertainty about abatement cost parameters, the variation in emission endowments, and the number of Member States.
\end{abstract}

Keywords Decentralization · Emissions trading - European Union · Pollution abatement · Private information

\section{Introduction}

The European Union Emissions Trading System (EU ETS) is the second large scale policy application of pollution permit markets, following the US sulfur permit market. As described by Kruger et al. (2007) and Böhringer et al. (2005), the structures of the two markets differ significantly. In the EU ETS, the supply of carbon dioxide permits is determined in a decentralized manner. Each Member State allocates an endowment of emissions to the permit market. The sum of these allocations over all Member States yields the supply. In the US sulfur permit market, the supply is determined by a single regulatory authority.

D. A. Malueg (ه)

Department of Economics, University of California, Riverside, CA 92521, USA

e-mail: david.malueg@ucr.edu

\section{A. J. Yates}

Department of Economics, E. Claiborne Robins School of Business, University of Richmond,

1 Gateway Rd, Richmond, VA 23173, USA

e-mail: ayates2@ richmond.edu 
Kruger et al. (2007) ask whether a similar arrangement might improve the performance of the EU ETS: rather than having each Member State allocate emissions, might it be better to have the EU central government make these decisions? The question is particularly relevant given that the EU's recent proposal for subsequent periods of the EU ETS does indeed recommend a centralized determination of permit supply (Commission of the European Communities 2008). Clearly there is an opportunity for economic analysis to inform the debate.

In this paper we identify some advantages and disadvantages of such centralized decision making. We construct a formal model containing these elements and delineate conditions under which centralization is preferred to the current decentralized structure of the EU ETS. Although political economy considerations such as "competitiveness" between emission sources in various Member States may play a role in actual policy deliberations, we abstract from such issues here and focus solely on Member States' costs of abating emissions.

It is natural to consider two abatement cost functions for each Member State because the EU ETS divides the sources of carbon emissions into two sectors, trading and non-trading. Each Member State has a fixed endowment of carbon emissions (the sum of which is equal to the EU-wide Kyoto Protocol target). A Member State allocates its endowment between the two sectors for sources within its border. The amount of emissions allocated to the nontrading sector directly determines the abatement costs for that sector. But the amount of emissions allocated to the trading sector, aggregated over all Member States, determines the supply of permits in the EU ETS. After the supply has been determined, the market opens and individual trading-sector sources in the various Member States may buy and sell permits. These market actions determine the abatement costs in the trading sector.

We analyze the interplay between behavior, information, and decentralization. We assume the EU central authority seeks to minimize total abatement costs, subject to the constraints on the Member States' emission endowments. In contrast, we assume each Member State is concerned with only its own abatement costs. Moreover, because there are relatively few Member States, a given Member State may act strategically when considering the amount of emissions to allocate to the trading sector. These considerations offer an advantage to centralization. Alternatively, one of the main tenets in the fiscal federalism literature is that local governments "possess knowledge of both local preferences and cost conditions that a central agency is unlikely to have" (Oates 1999, p 1123). Applying this insight to the EU ETS suggests that the Member States have better information about their own abatement costs than does the EU central authority. This consideration offers an advantage to decentralization. The information of the Member States themselves in regard to the abatement costs of other Member States is yet another consideration. While perhaps not as obvious as the others, we will see the consideration that abatement costs are private information offers an advantage to centralization.

Following Vives (2002), we consider four combinations of behavior and information for the Member States. In each case we compare the total abatement costs of the existing decentralized system to an alternative centralized system. When behavior is strategic, the Member States account for the fact that their allocations to the trading sector subsequently influence the price in the permit market. Thus, a Member State may want to manipulate the price to lower its abatement costs. When behavior is nonstrategic, the Member States do not account for the effect of their allocations on the permit price. Regardless of the behavior of the Member States, we assume that the permit market itself is competitive, given the supply of permits. This is because the market consists of over 12,000 sources, so no single source has market power. The information of the Member States about the other Member States' abatement costs gives rise to our two information structures. When there is full information, 
the abatement costs are common knowledge among the Member States. When there is private-information, the abatement costs of a Member State are private information to that state.

Because aggregate carbon emissions are fixed by the Kyoto Protocol, cost-effectiveness is the appropriate first-best welfare criterion for evaluating the EU ETS. In the cost-effective solution, total abatement costs are minimized subject to the constraint that total emissions equal the Kyoto protocol target. Our benchmark case consists of full information and nonstrategic behavior: under fairly general conditions, we show that the decentralized system is cost-effective and so is clearly preferred to the centralized system. Although this result offers support for the current design of the EU ETS, one might argue that it is unrealistic to assume that Member States will behave nonstrategically and that they have full information about abatement costs. To see the effects of relaxing these assumptions, we restrict the model to quadratic abatement costs and determine whether the decentralized system or the centralized system leads to lower total abatement costs. Private information increases the relative costs of decentralization, but as long as Member States are nonstrategic, decentralization is still preferred. Strategic behavior also increases the relative costs of decentralization, and in fact this increase is large enough to make the choice between centralization and decentralization ambiguous. With strategic behavior, it is possible that the centralization leads to lower costs, depending on the number of Member States, the degree of uncertainty about abatement cost parameters, and the variation in emission endowments.

It is interesting to interpret our results in the context of the EU principle of subsidiarity, a legal principle designed to be a check on central authority. According to this principle, actions should generally be taken at the Member State level, unless actions at the EU level would be more effective. Our results identify conditions under which the centralized system leads to lower expected total costs that the decentralized system, and hence actions at the EU level may be appropriate.

\section{The Models and a Benchmark Solution}

\subsection{The General Model}

Let $W$ be the EU-wide Kyoto protocol target level of emissions. Because this is fixed, we need only consider abatement cost functions, not damage from emissions. There are $m$ Member States, where $m \geq 2$. Let $w_{i}$ be the Kyoto endowment of emissions for Member State $i$, with $\sum w_{i}=W$. The average endowment is $\bar{w}=W / m$ and the variance of the endowment is $\operatorname{var}(w)=(1 / m) \sum\left(w_{i}-\bar{w}\right)^{2}$. The two sectors in each Member State are summarized by their abatement cost functions. Let $c_{i}^{t}\left(t_{i}\right)$ be the abatement cost in the trading sector of Member State $i$ as a function of emissions of the trading sector $t_{i}$; let $c_{i}^{n}\left(n_{i}\right)$ be the abatement cost in the non-trading sector as a function of emissions of the non-trading sector $n_{i}$. We refer to $-\partial c_{i}^{j}(x) / \partial x$ as the marginal abatement cost in sector $j$ of Member State $i$, where $i \in\{1, \ldots, m\}$ and $j \in\{t, n\}$. The minus sign arises because increases in abatement correspond to reductions in emissions. As is standard, we assume that marginal abatement cost functions are positive decreasing functions of emissions.

We define an emissions profile $\left(t_{i}, n_{i}\right)_{i=1}^{m}$ to be cost-effective if it minimizes aggregate abatement costs, subject to the constraint that total emissions equal $W$. A planner with full information about abatement costs would solve 


$$
\begin{aligned}
& \min _{\left(t_{i}, n_{i}\right)} \sum\left(c_{i}^{t}\left(t_{i}\right)+c_{i}^{n}\left(n_{i}\right)\right) \\
& \text { such that } \sum\left(t_{i}+n_{i}\right)=W .
\end{aligned}
$$

The first-order conditions imply that the marginal abatement costs are equal across sectors within a Member State and also equal across all Member States. Let the solution to this problem be denoted by $\left(t_{i}^{*}, n_{i}^{*}\right)_{i=1}^{m}$.

Our information structures are drawn from the Industrial Organization literature, where it is sometimes assumed that rival firms know each others cost parameters, but a government regulator does not (see for example, Bernheim and Whinston 1986). In our model, Member States correspond to rival firms and the EU central authority corresponds to the government regulator. So we initially assume that, in the full-information case, Member States have better information than the EU central authority about other Member States' abatement costs. Alternatively, one might argue that when the Member States know each others' abatement costs, the EU central authority knows them as well. In Sect.3.6 we present a complete analysis of the model under this alternative assumption.

\subsection{The Decentralized Solution}

Now consider the decentralized problem facing each Member State. A Member State must decide how to allocate its Kyoto endowment of emissions, $w_{i}$, between the trading and nontrading sectors. Suppose that Member State $i$ allocates emissions $e_{i}$ to the trading sector. It immediately follows that emissions of the non-trading sector are $w_{i}-e_{i}$. We refer to the relation $e_{i}+n_{i}=w_{i}$ as the emission-endowment constraint. Emissions of the trading sector, $t_{i}$, will not generally equal $e_{i}$, however, as sources in the trading sector will buy and sell permits. The exact form for a Member State's decentralized problem will depend on the various combinations of information and behavior.

Member States may or may not account for how their choice of $e_{i}$ influences the permit price. But, because many sources buy and sell permits, we assume that, the permit market itself is competitive once the sector allocations have been specified. It is well known that a competitive permit market equilibrium minimizes aggregate abatement costs. A convenient way to characterize the equilibrium uses the aggregate abatement cost function for the trading sector, defined as

$$
\begin{aligned}
& C^{t}(E) \equiv \min _{t_{i}} \sum c_{i}^{t}\left(t_{i}\right) \\
& \text { such that } \sum t_{i}=E
\end{aligned}
$$

where $E$, the supply of permits, equals $\sum e_{i}$. The Lagrange multiplier for this problem corresponds to the equilibrium price of permits $p$. So the first-order condition for $t_{i}$ can be written

$$
-\frac{\partial c_{i}^{t}\left(t_{i}\right)}{\partial t_{i}}=p,
$$

the familiar result that marginal abatement cost equals the permit price. Equation (3) defines the trading-sector constraint. It delineates how the emissions of the trading sector are determined by the market price of permits.

Because we assume the permit market is competitive, the variable $t_{i}$ is not a choice variable for the Member States. Given the price of permits (which will indeed depend on the choice variables $e_{i}$ ), the variable $t_{i}$ is completely determined by the trading sector constraint, 
and in turn $t_{i}$ determines the trading sector abatement costs. For all the cases we consider, then, a strategy for each member state is simply a choice of $e_{i}$.

\subsection{A Decentralization Benchmark: Full Information and Nonstrategic Behavior}

Consider first the combination of full information and nonstrategic behavior. Member State $i$ selects $e_{i}$ to minimize the sum of abatement costs and permit expenditures, subject to the trading-sector constraint and the emission-endowment constraint. So Member State $i$ solves

$$
\begin{gathered}
\min _{e_{i}} c_{i}^{t}\left(t_{i}\right)+c_{i}^{n}\left(n_{i}\right)+p\left(t_{i}-e_{i}\right) \\
\text { such that }-\frac{\partial c_{i}^{t}\left(t_{i}\right)}{\partial t_{i}}=p \\
e_{i}+n_{i}=w_{i} \\
e_{i} \geq 0 n_{i} \geq 0 .
\end{gathered}
$$

Because the Member State does not behave strategically (i.e., it does not account for the effect of its decisions on the market price of permits) the trading-sector constraint is not a function of $e_{i}$ and hence is superfluous for determining $e_{i}$. Substituting the emission-endowment constraint into the objective function (4) yields a problem in terms of the single variable $e_{i}$. The first-order condition for an interior solution requires that the marginal abatement cost in the non-trading sector equals the price of permits.

Even though a Member State does not account for the effect of its decisions on the market price of permits, it does account for the choices of the other Member States. The solution to the decentralized system is thus described by a Nash equilibrium. A profile of allocations to the trading sector is a Nash equilibrium if $e_{i}$ solves (4) for each $i$. To determine this equilibrium, one solves the $m$ first-order conditions for the $m$ choice variables $e_{i}$. At first blush, it may seem difficult to solve for the equilibrium because the price appears in the first-order conditions. By solving (2), we can determine the market price as a function of $\sum e_{i}$, so the first-order conditions can be written exclusively in terms of the variables $e_{i}$. Given the Nash equilibrium values $e_{i}^{d}$, the values for $t_{i}^{d}$ and $n_{i}^{d}$ are determined by the trading-sector and emission endowment constraints (the superscript $d$ denotes "decentralized").

Our first main result gives conditions under which the decentralized solution is cost-effective. The proof is in the Appendix.

Proposition 1 Suppose that the Member States have full information and do not behave strategically. If $w_{i}>n_{i}^{*}$ for each $i$, then the decentralized solution is cost-effective.

Consider the intuition for Proposition 1. Each Member State sets the marginal abatement cost in the non-trading sector equal to the market price of permits. In the equilibrium of the permit market, the trading sector constraint implies that marginal abatement costs in the trading sector equal the market price of permits. Thus, marginal abatement costs are equal across both sectors within a Member State and also equal across all Member States. This is the condition for cost-effectiveness.

In subsequent sections, we analyze what happens when Member States do not have full information (hence the market price is uncertain) or they behave strategically (hence they account for the influence of their choice of $e_{i}$ on the market price). 


\subsection{The Centralized Solution}

In the centralized solution, the EU central authority determines how the Kyoto endowment of emissions is allocated between the trading and non-trading sectors for each Member State. The sum of the allocations of emissions to the trading sector yields the supply of permits. Then the permit market opens and the emissions of the trading sectors across Member States are determined by the market. Hence the emissions of the trading sector, $t_{i}$, for a given Member State will generally be different from the allocation of emissions, $e_{i}$, assigned by the EU central authority to that Member State.

The EU central authority's problem is

$$
\begin{gathered}
\min _{e_{i}} \mathcal{E}\left[C^{t}\left(\sum e_{i}\right)\right]+\sum \mathcal{E}\left[c_{i}^{n}\left(n_{i}\right)\right] \\
\text { such that } e_{i}+n_{i}=w_{i}, \quad i=1, \ldots, m, \\
e_{i} \geq 0 \quad n_{i} \geq 0,
\end{gathered}
$$

where $\mathcal{E}[\cdot]$ denotes the expectation operator. Substituting the constraints into the objective function yields a problem in terms of the $m$ choice variables $e_{i}$. The first-order condition for an interior $e_{i}$ is

$$
\mathcal{E}\left[\frac{\partial C^{t}}{\partial E}\right]-\mathcal{E}\left[\frac{\partial c_{i}^{n}}{\partial n_{i}}\right]=0 .
$$

By the Envelope Theorem, the first term is equal to the negative of the expected market price. So the first-order condition indicates that expected price is equal to the expected marginal abatement costs in the non-trading sector.

The centralized solution is described by the quantities $e_{i}^{c}, n_{i}^{c}$, and $t_{i}^{c}$ (the superscript $c$ denotes "centralized"). The values for $e_{i}^{c}$ are found by solving the system of $m$ equations specified by (5). The values for $n_{i}^{c}$ follow from the emission-endowment constraint $e_{i}+n_{i}=w_{i}$. In the trading sector, firms will purchase permits until the marginal abatement costs equal price. Thus the values for $t_{i}^{c}$ follow from the trading-sector constraint (3). See the Appendix for details of these calculations.

\section{Centralization Versus Decentralization: The Roles of Private Information and Strategic Behavior}

We now compare the centralized solution to the various decentralized solutions. To determine a given decentralized solution, we first solve a system of first-order conditions for the Member States' allocations of emissions to the trading sector (as the Nash equilibrium is defined with respect to these variables). The allocation of emissions to the non-trading sector and the emissions of the trading sector then follow by the emission-endowment and trading-sector constraints.

To obtain an analytical solution, we consider a special case of quadratic abatement costs. Let the non-trading sector abatement costs be given by

$$
c_{i}^{n}\left(n_{i}\right)=A_{i}-\gamma_{i} n_{i}+(\mu / 2)\left(n_{i}\right)^{2},
$$

and let the trading sector abatement costs be given by

$$
c_{i}^{t}\left(t_{i}\right)=D_{i}-\theta_{i} t_{i}+(\lambda / 2)\left(t_{i}\right)^{2} .
$$


The parameters $A_{i}$ and $D_{i}$ simply define the cost of obtaining zero emissions and they do not play a role in our analysis. The relevant heterogeneity between Member States is captured solely by the parameters $\gamma_{i}$ and $\theta_{i}$. The parameters $\mu$ and $\lambda$ are common knowledge to all Member States and the EU central authority. In the absence of any regulation, the sectors would emit $\gamma_{i} / \mu$ and $\theta_{i} / \lambda$, where the marginal benefit (to firms) of additional emissions is zero. These are commonly called the "business-as-usual" levels of emissions. By substituting the quadratic cost functions into (2) and solving for the Lagrange multiplier, we see that the equilibrium price of permits is

$$
p=\bar{\theta}-\lambda \bar{e},
$$

where $\bar{e}=(1 / m) \sum e_{i}$ is the average permit supply.

In the quadratic-cost model, our two information structures relate to the parameters $\gamma_{i}$ and $\theta_{i}$. We consider cases in which the Member States have full information or private information about these parameters. When there is full information, the $\gamma_{i}$ 's and $\theta_{i}$ 's are common knowledge among the Member States. When there is private information, Member State $i$ knows the value of its own parameters, but considers $\gamma_{j}$ to be a random variable with expected value $\tilde{\gamma}$ and variance $\sigma_{\gamma}^{2}$, and considers $\theta_{j}$ to be a random variable with expected value $\tilde{\theta}$ and variance $\sigma_{\theta}^{2}$. A Bayesian-Nash equilibrium is used to characterize the decentralized solution in the private-information case. In either case, the Member States have an informational advantage over the EU central authority. (We will relax this assumption in Sect.3.6.) The EU central authority does not know the true values for the $\gamma_{i}$ 's and considers them to be random variables with expected value $\tilde{\gamma}$ and variance $\sigma_{\gamma}^{2}$. Likewise, the EU central authority considers the $\theta_{i}$ 's to be random variables with expected value $\tilde{\theta}$ and variance $\sigma_{\theta}^{2}$. We assume all the random variables are independent of each other.

We start with the full-information, nonstrategic case. From Proposition 1, we know that decentralization is better than centralization in this case, but it is instructive to show this for the quadratic abatement cost functions before tackling the private-information and strategic cases.

\subsection{The Full-Information, Nonstrategic Case}

As discussed above, in this case each Member State $i$ solves (4). The solution for $e_{i}$ is described by the first-order condition

$$
-\frac{\partial c_{i}^{n}\left(w_{i}-e_{i}\right)}{\partial n_{i}}=p .
$$

With the quadratic abatement cost functions, this becomes

$$
\gamma_{i}-\mu\left(w_{i}-e_{i}\right)=p
$$

Using the expression for $p$ from (6), we can solve the $m$ equations from (7) for the Nash equilibrium quantities $e_{i}^{d}$. The values for $t_{i}^{d}$ are found from the trading-sector constraint (3). Finally, the values for $n_{i}^{d}$ are determined by the emission-endowment constraint $e_{i}+n_{i}=w_{i}$. See the Appendix for details of these calculations.

We can now compare the expected total costs of the decentralized solution, $\mathcal{C}^{d}$, to the expected total costs of the centralization solution, $\mathcal{C}^{c}$. We have

$$
\mathcal{C}^{d}=\sum \mathcal{E}\left[c_{i}^{t}\left(t_{i}^{d}\right)\right]+\sum \mathcal{E}\left[c_{i}^{n}\left(n_{i}^{d}\right)\right]
$$


and

$$
\mathcal{C}^{c}=\sum \mathcal{E}\left[c_{i}^{t}\left(t_{i}^{c}\right)\right]+\sum \mathcal{E}\left[c_{i}^{n}\left(n_{i}^{c}\right)\right] .
$$

In the Appendix, we show that the difference between expected total costs is

$$
\mathcal{C}^{d}-\mathcal{C}^{c}=-\frac{\sigma_{\gamma}^{2}}{2 \mu}\left(m-\frac{\lambda}{\mu+\lambda}\right)-\frac{\sigma_{\theta}^{2}}{2(\mu+\lambda)} .
$$

As expected from Proposition 1, this expression is negative, implying that decentralization leads to lower expected total costs than centralization. As the number of Member States increases, the per Member State cost advantage of decentralization increases, and in the limit is $\frac{1}{2 \mu} \sigma_{\gamma}^{2}$.

Turning now to the other cases, we first describe the decentralized solution for each case. In particular, we determine the first-order conditions for $e_{i}^{d}$. Rather than discussing the comparison to the centralized solution on a case by case basis, we wait until all the cases have been described and then make the comparisons all at once.

\subsection{The Full-Information, Strategic Case}

In this case, we continue to assume that Member States have full information about the $\gamma_{i}$ 's and $\theta_{i}$ 's, but they $d o$ account for the effect of $e_{i}$ on the price of permits. Recall from (6) that the market price of permits is $\bar{\theta}-\lambda \bar{e}$. Thus, Member State $i$ solves

$$
\begin{gathered}
\min _{e_{i}} c_{i}^{t}\left(t_{i}\right)+c_{i}^{n}\left(n_{i}\right)+(\bar{\theta}-\lambda \bar{e})\left(t_{i}-e_{i}\right) \\
\text { such that } \theta_{i}-\lambda t_{i}=\bar{\theta}-\lambda \bar{e} \\
\qquad e_{i}+n_{i}=w_{i} \\
e_{i} \geq 0 \quad n_{i} \geq 0 .
\end{gathered}
$$

The trading-sector constraint (9) is now a critical part of the Member State's problem. The Member State realizes that the choice of $e_{i}$ influences the price of permits, which determines the emissions of the trading sector, which in turn determines trading sector abatement costs. Substituting the trading-sector and emission endowment constraints into the objective function yields a problem in terms of a single variable $e_{i}$. The first-order condition for an interior solution is

$$
\gamma_{i}-\mu\left(w_{i}-e_{i}\right)-(\bar{\theta}-\lambda \bar{e})-(1 / m)\left(\theta_{i}-\bar{\theta}+\lambda \bar{e}\right)+(\lambda / m) e_{i}=0 .
$$

The Nash equilibrium is found by solving the set of $m$ equations corresponding to (10).

To interpret the behavior of the Member States in this case, we use (9) to rewrite (10) as

$$
\underbrace{\gamma_{i}-\mu\left(w_{i}-e_{i}\right)}_{M C=-\partial c_{i}^{n} / \partial n_{i}}-\underbrace{\left((\bar{\theta}-\lambda \bar{e})-(\lambda / m) e_{i}\right)}_{M R}-(\lambda / m) t_{i}=0 .
$$

The term labeled MC is analogous to the marginal cost of production for a traditional Cournot oligopolist. (For a Member State, the cost of "producing" $e_{i}$ is that non-trading sector emissions must be reduced by an equivalent amount.) The term labeled MR is analogous to the marginal revenue for a traditional oligopolist facing demand curve $p(\bar{e})=\bar{\theta}-\lambda \bar{e}$. Suppose for the moment that a given Member State did not have any sources in the trading sector. Then such a Member State would indeed act as a traditional oligopolist and set 


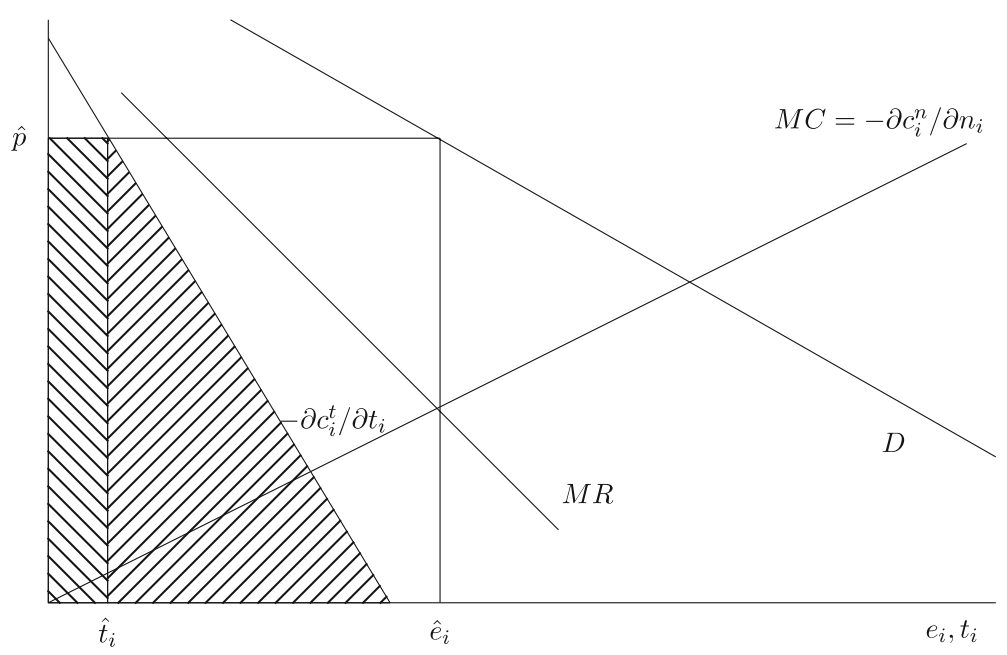

Fig. 1 Strategic behavior

MC equal to MR so that "profit" from permit sales is maximized. Now return to our general model in which the Member State does have sources in the trading sector. Intuitively, the Member State behaves differently from a Cournot oligopolist because it accounts for the fact that some of the demand for permits comes from its own trading sector. Indeed, Eq. (11) shows that, in addition to marginal cost and marginal revenue, there is an additional term $(\lambda / m) t_{i}$ due to the trading sector. So marginal cost exceeds marginal revenue at the optimal $e_{i}$.

Figure 1 gives a graphical explanation of (11), particularly the source of the $(\lambda / m) t_{i}$ term. Starting with a traditional Cournot oligopoly diagram (consisting of $M C, D$, and $M R$ curves) we have added the Member State's marginal abatement cost in the trading sector. The marginal cost curve-equal to the marginal abatement cost in the non-trading sector-slopes upward because it is presented as a function of $e_{i}$ rather than $n_{i}$ (reflecting the emission-endowment constraint). Conversely, the marginal abatement cost in the trading sector slopes downward because it is presented directly in terms of $t_{i}$. The demand curve is equal to the horizontal sum of all Member States' non-trading sector marginal abatement cost curves and hence lies above the non-trading sector marginal abatement cost curve of the individual Member State. Suppose that the Member State initially selects an endowment of emissions $\hat{e}_{i}$ such that marginal revenue is equal to marginal cost. This yields a market price for permits $\hat{p}$ and trading sector emissions $\hat{t}_{i}$ (where price is equal to marginal abatement cost). At this point, profit from permit sales is maximized, but we must also account for total expenditures in the trading sector. Total expenditures include permit purchases (the striped rectangle) and abatement cost (the striped triangle). Now consider a small increase in the endowment of emissions from $\hat{e}_{i}$ to $\hat{e}_{i}+\Delta e_{i}$. From the demand curve, this leads to a change in price of $\Delta p=-(\lambda / m) \Delta e_{i}$. The first-order change in total expenditures in the trading sector is $\hat{t}_{i} * \Delta p=-(\lambda / m) \hat{t}_{i} \Delta e_{i}$. This dominates the first-order change in profit from permit sales (which is zero), so the welfare of the Member State increases. From (11), we see that the Member State should continue to increase $\hat{e}_{i}$ until the loss in profit on permit sales is just equal to the reduction in trading sector expenditures. 


\subsection{The Private-Information, Nonstrategic Case}

In this case, the parameters $\gamma_{i}$ and $\theta_{i}$ are private information to Member State $i$. Thus, a Bayesian-Nash equilibrium is the appropriate solution concept. Member State $i$ solves

$$
\begin{gathered}
\min _{e_{i}} \mathcal{E}\left[c_{i}^{t}\left(t_{i}\right)+c_{i}^{n}\left(n_{i}\right)+p\left(t_{i}-e_{i}\right) \mid \gamma_{i}, \theta_{i}\right] \\
\text { such that } \theta_{i}-\lambda t_{i}=p \\
\qquad e_{i}+n_{i}=w_{i} \\
e_{i} \geq 0 \quad n_{i} \geq 0 .
\end{gathered}
$$

As with the other nonstrategic case, the trading-sector constraint (13) is not needed to solve for $e_{i}$. After substituting the emission-endowment constraint into the objective function, the first-order condition for an interior solution is

$$
\gamma_{i}-\mu\left(w_{i}-e_{i}\right)-\mathcal{E}\left[p \mid \gamma_{i}, \theta_{i}\right]=0 .
$$

In the non-trading sector the marginal abatement cost is equal to the expected price for permits. The Bayesian-Nash equilibrium is found by solving the set of $m$ equations corresponding to (14).

\subsection{The Private-Information, Strategic Case}

In the final case, Member States account for the effect of $e_{i}$ on price and also have private information about abatement costs. Member State $i$ solves

$$
\begin{aligned}
& \min _{e_{i}} \mathcal{E}\left[c_{i}^{t}\left(t_{i}\right)+c_{i}^{n}\left(n_{i}\right)+(\bar{\theta}-\lambda \bar{e})\left(t_{i}-e_{i}\right) \mid \gamma_{i}, \theta_{i}\right] \\
& \text { such that } \theta_{i}-\lambda t_{i}=(\bar{\theta}-\lambda \bar{e}) \\
& \qquad e_{i}+n_{i}=w_{i} \\
& e_{i} \geq 0 \quad n_{i} \geq 0 .
\end{aligned}
$$

Following the other strategic case, we substitute the emission-endowment and trading-sector constraints into the objective function. The first-order condition is

$$
\gamma_{i}-\mu\left(w_{i}-e_{i}\right)-\mathcal{E}\left[(\bar{\theta}-\lambda \bar{e})+(1 / m)\left(\theta_{i}-\bar{\theta}+\lambda \bar{e}\right)-(\lambda / m) e_{i} \mid \gamma_{i}, \theta_{i}\right]=0 .
$$

The Bayesian-Nash equilibrium is found by solving the set of $m$ equations corresponding to (17).

\subsection{Comparisons}

We now make the centralization versus decentralization comparisons, following the template of the full-information, nonstrategic case. Solving the appropriate system of first-order equations for $e_{i}^{d}$ yields the (Bayesian) Nash equilibrium. The constraints determine $t_{i}^{d}$ and $n_{i}^{d}$, from which the cost difference $\mathcal{C}^{d}-\mathcal{C}^{c}$ can be calculated. We provide details of these calculations in the Appendix. The results, including the full-information, nonstrategic case for reference, are shown in Table 1. If $\mathcal{C}^{d}-\mathcal{C}^{c}$ is negative, then decentralization leads to lower expected total costs than centralization.

The results in Table 1 enable us to isolate the effects of private information and behavior. Consider, for example, the first and third rows. The difference between $\mathcal{C}^{d}-\mathcal{C}^{c}$ in these rows gives the effect of moving from full information to private information, keeping behavior 
Table 1 Summary of results

\begin{tabular}{ll}
\hline Case & $\mathcal{C}^{d}-\mathcal{C}^{c}$ \\
\hline $\begin{array}{l}\text { Full-Info } \\
\text { Nonstrategic }\end{array}$ & $-\frac{\sigma_{\gamma}^{2}}{2 \mu}\left(m-\frac{\lambda}{\mu+\lambda}\right)-\frac{\sigma_{\theta}^{2}}{2(\mu+\lambda)}$ \\
$\begin{array}{l}\text { Full-Info } \\
\text { Strategic }\end{array}$ & $\frac{m \mu \lambda^{2}}{2(\mu m+\lambda)^{2}} \operatorname{var}(w)-\sigma_{\gamma}^{2}\left((m-1) \frac{\frac{m^{2} \mu}{2}+m \lambda}{(m \mu+\lambda)^{2}}+\frac{1}{2(\mu+\lambda)}\right)$ \\
& $-\frac{\sigma_{\theta}^{2}}{2(m \mu+\lambda)^{2}(\mu+\lambda)}\left(\mu^{2}\left(m^{2}-m+1\right)+(m+1) \lambda \mu+\lambda^{2}\right)$ \\
Private-Info & $-\frac{\sigma_{\gamma}^{2} m^{2}}{2(m \mu+\lambda)}-\frac{\sigma_{\theta}^{2}}{2(m \mu+\lambda)}$ \\
Nonstrategic & $\frac{m \mu \lambda^{2}}{2(\mu m+\lambda)^{2}} \operatorname{var}(w)-\sigma_{\gamma}^{2} m^{3} \frac{\left((3 m-2) \lambda+m^{2} \mu\right)}{2\left(m^{2} \mu+\lambda(2 m-1)\right)^{2}}-\sigma_{\theta}^{2}(2 m-1) \frac{((2 m-1) \lambda+m \mu)}{2\left(m^{2} \mu+\lambda(2 m-1)\right)^{2}}$ \\
Private-Info & Strategic
\end{tabular}

constant at nonstrategic. This and similar calculations yield the following proposition. (The proof is straightforward but algebraically tedious.)

Proposition 2 Keeping behavior constant, moving from full information to private information increases $\mathcal{C}^{d}-\mathcal{C}^{c}$. Keeping information constant, moving from nonstrategic behavior to strategic behavior also increases $\mathcal{C}^{d}-\mathcal{C}^{c}$.

Proposition 2 shows that the difference in expected total costs is greater when the Member States have private information than when they have full information. Thus private information confers an advantage to centralization. Likewise, the difference in expected total costs is greater when the Member States behave strategically than when they behave nonstrategically. Thus, strategic behavior also confers an advantage to centralization.

Turning now to the question of whether the current structure of the EU ETS can be improved by centralization, the following proposition follows directly from Table 1 .

Proposition 3 If Member States are nonstrategic, then $\mathcal{C}^{d}-\mathcal{C}^{c}<0$.

Proposition 3 shows that decentralization leads to lower expected total costs when Members States are nonstrategic, regardless of whether information is full or private. (Recall that Member States have an informational advantage over the central authority.) Combining the results of Propositions 2 and 3 shows that, if Member States are nonstrategic, then even though private information makes centralization more appealing, the increase in the cost difference is not enough to make centralization the better choice.

If Member States are strategic, then the sign of $\mathcal{C}^{d}-\mathcal{C}^{c}$ is ambiguous for both full and private information. For these cases, $\mathcal{C}^{d}-\mathcal{C}^{c}$ depends on three terms (see Table 1). The first term is positive and the other terms are negative, implying that either decentralization or centralization may lead to lower total expected costs, depending on the values of the parameters $\sigma_{\gamma}^{2}, \sigma_{\theta}^{2}, \operatorname{var}(w)$, and $m$. The effects of the informational parameters are straightforward. Suppose that $\sigma_{\gamma}^{2}$, the variance of the EU central authority's uncertainty about non-trading sector abatement costs, increases. Then the magnitude of the second term increases, which makes it more likely that decentralization is preferred. A similar result holds for $\sigma_{\theta}^{2}$, the 
variance of the EU central authority's uncertainty about trading sector abatement costs. As both variances go to zero, centralization is preferred.

Now consider the variance of the Kyoto emission endowments. If $\operatorname{var}(w)$ increases, then the magnitude of the first term increases, which makes it more likely that centralization is preferred. If $\operatorname{var}(w)$ goes to zero, then decentralization is preferred. We see that the ability of the Member States to manipulate the permit market is related to the distribution of the Kyoto emission endowments. In actual practice, there is wide variation in the Kyoto emission endowments (see Convery and Redmond 2007) and hence we might expect a significant degree of strategic behavior. Although he considers a different market structure, Hahn (1984) also finds that the distribution of the emission endowments plays a critical role in determining the degree of market power.

The final parameter is $m$, the number of Member States. In its current form, the EU has 27 member states. Simple intuition suggests that as the number of Member States increases, the ability of a given Member State to manipulate the market to its advantage decreases. So for large values of $m$, the decentralized system should lead to lower total expected costs. The effect of an increase in $m$ on the first term is ambiguous. Suppose for the moment, though, that $\operatorname{var}(w)$ is kept constant with respect to $m$. As $m$ increases, the magnitude of the first term decreases to zero and the magnitude of the second term increases. So for large enough $m$, the cost difference must be negative, confirming our intuition that only for small values of $m$ is it possible that centralization is preferred to decentralization. This result continues to hold even if $\operatorname{var}(w)$ "modestly" increases in $m$.

\subsection{Alternative Informational Assumptions}

As discussed above, one might argue that when the Member States have full information, then the EU central authority has full information as well. It is easy to adapt our analysis to account for this possibility. Suppose that ex ante, the $\gamma_{i}$ 's and $\theta_{i}$ 's are random variables, and when there is full information, these parameters are revealed to both the EU and the Member States before they make their decisions. When there is private information, $\gamma_{i}$ and $\theta_{i}$ are only revealed to Member State $i$. The analysis of private information is thus the same as before, only the full-information cases require a new calculation. In other words, the entries in the first and second rows of Table 1 would change, but the entries in the third and fourth rows would not.

For full information, then, we first determine a new centralized solution, and then compare this solution to the decentralized solution for the two types of behavior. It turns out that the new centralized solution is exactly the same as the decentralized full-information, nonstrategic solution. So the new entry in the first row of Table 1 would be zero. The new entry in the second row, reflecting the ex ante value of $\mathcal{C}^{d}-\mathcal{C}^{c}$ for the full-information, strategic case, would be

$$
\frac{m \mu \lambda^{2}}{2(\mu m+\lambda)^{2}} \operatorname{var}(w)+\sigma_{\gamma}^{2}(m-1) \frac{\lambda^{2}}{2 \mu(\mu m+\lambda)^{2}}+\sigma_{\theta}^{2}(m-1) \frac{\mu}{2(\mu m+\lambda)^{2}} .
$$

This expression is positive, because in this case, the Member States do not have an informational advantage over the EU central authority and they behave strategically. So clearly centralization is preferred.

The main effect of these changes is on the role of private information. Keeping behavior constant, moving from full information to private information now decreases $\mathcal{C}^{d}-\mathcal{C}^{c}$. So private information confers an advantage to decentralization. This is opposite to Proposition 2. Why the difference? Proposition 2 identifies the effect of Member States' moving from full 
information to private information, keeping the information of the EU central authority fixed. The new result identifies the effect of both the Member States and the EU central authority moving from full information to private information. Member States' moving to private information confers an advantage to centralization, but this effect is overwhelmed when the EU central authority moves to private information as well.

\section{Conclusion}

Our findings offer guidance for the design of the EU permit market. Given the size and prominence of this market, it will no doubt be a model for other regional permit systems. So our findings will likely apply to them as well. The performance of a decentralized permit system depends critically on whether the individual Member States behave strategically. If they do not, then, within the context of our model, the permit market should be decentralized. If they do, then the centralization versus decentralization question depends on the number of Member States, the degree of uncertainty about abatement cost parameters, and the variance of the Kyoto emission endowments.

For analytical clarity, we have analyzed the choice between two extreme systems. In the decentralized system, the choice of permit endowment is determined solely by the Member States, and in the centralized system, the choice of permit endowment is determined solely by the EU central authority. In current practice, the EU ETS employs a modified form of the decentralized system, in which the choices of the Member States must be approved by the EU central authority. In a modified centralized system, these roles could be reversed. One may perhaps analyze systems of this type by employing a leader-follower framework. It is likely, however, that the parameters identified here will continue to play a critical role in the comparison between centralization and decentralization.

Acknowledgements We would like to thank several anonymous referees for helpful comments and suggestions.

Open Access This article is distributed under the terms of the Creative Commons Attribution Noncommercial License which permits any noncommercial use, distribution, and reproduction in any medium, provided the original author(s) and source are credited.

\section{Appendix}

Proof of Proposition 1 The first-order conditions for (1) define the first-best solution $t_{i}^{*}, n_{i}^{*}$. We have $\frac{\partial c_{i}^{t}\left(t_{i}^{*}\right)}{\partial t_{i}}=-\Lambda$ and $\frac{\partial c_{i}^{n}\left(n_{i}^{*}\right)}{\partial n_{i}}=-\Lambda$, where $\Lambda$ is the Lagrange multiplier for the constraint. The first-order condition for an interior solution to the decentralized problem (4) is $\frac{\partial c_{i}^{n}\left(w_{i}-e_{i}\right)}{\partial n_{i}}=-p$. By the trading-sector constraint, we also have $\frac{\partial c_{i}^{t}\left(t_{i}\right)}{\partial t_{i}}=-p$. Let the solution to these equations be given by $\hat{t}_{i}$ and $\hat{e}_{i}$; and let $\hat{n}_{i}=w_{i}-\hat{e}_{i}$. We need to show that $\hat{t}_{i}=t_{i}^{*}, \hat{n}_{i}=n_{i}^{*}$, and $\hat{e}_{i}>0$.

First note that in equilibrium, we have $\sum \hat{t}_{i}=\sum \hat{e}_{i}$. So

$$
\sum \hat{t}_{i}+\sum \hat{n}_{i}=\sum \hat{e}_{i}+\sum\left(w_{i}-\hat{e}_{i}\right)=\sum \hat{e}_{i}+\sum w_{i}-\sum \hat{e}_{i}=\sum w_{i}=W
$$

Next we show that $p=\Lambda$. Suppose instead that $p<\Lambda$. By the assumptions on the first and second derivatives of $c^{t}$ and $c^{n}$, we have $\hat{t}_{i}>t_{i}^{*}$ and $\hat{n}_{i}>n_{i}^{*}$. But then 
Table 2 Expectations

\begin{tabular}{lll}
\hline$\gamma$ & $\theta$ & $\gamma$ and $\theta$ \\
\hline $\mathcal{E}[\bar{\gamma}]=\tilde{\gamma}$ & $\mathcal{E}[\bar{\theta}]=\tilde{\theta}$ & $\mathcal{E}\left[\gamma_{i} \theta_{j}\right]=\tilde{\gamma} \tilde{\theta}$ \\
$\mathcal{E}\left[\bar{\gamma}^{2}\right]=\sigma_{\gamma}^{2} / m+\tilde{\gamma}^{2}$ & $\mathcal{E}\left[\bar{\theta}^{2}\right]=\sigma_{\theta}^{2} / m+\tilde{\theta}^{2}$ & $\mathcal{E}[\bar{\gamma} \bar{\theta}]=\tilde{\gamma} \tilde{\theta}$ \\
$\mathcal{E}\left[\gamma_{i} \bar{\gamma}\right]=\sigma_{\gamma}^{2} / m+\tilde{\gamma}^{2}$ & $\mathcal{E}\left[\theta_{i} \bar{\theta}\right]=\sigma_{\theta}^{2} / m+\tilde{\theta}^{2}$ & $\mathcal{E}\left[\gamma_{i} \bar{\theta}\right]=\tilde{\gamma} \tilde{\theta}$ \\
$\mathcal{E}\left[S_{1}\right]=0$ & $\mathcal{E}\left[S_{2}\right]=0$ & $\mathcal{E}\left[\theta_{i} \bar{\gamma}\right]=\tilde{\gamma} \tilde{\theta}$ \\
$\mathcal{E}\left[\left(S_{1}\right)^{2}\right]=m \sigma_{\gamma}^{2}$ & $\mathcal{E}\left[\left(S_{2}\right)^{2}\right]=m \sigma_{\theta}^{2}$ & $\mathcal{E}\left[S_{1} S_{2}\right]=0$ \\
& $\mathcal{E}\left[S_{2} \bar{\theta}\right]=\sigma_{\theta}^{2}$ & $\mathcal{E}\left[S_{1} \bar{\theta}\right]=0$ \\
\hline
\end{tabular}

$$
\sum\left(\hat{t}_{i}+\hat{n}_{i}\right)>\sum\left(t_{i}^{*}+n_{i}^{*}\right)=W,
$$

a contradiction. Likewise, if $p>\Lambda$, we get a contradiction. Thus, $p=\Lambda$ and hence $\hat{t}_{i}=t_{i}^{*}$ and $\hat{n}_{i}=n_{i}^{*}$. It follows that $\hat{e}_{i}=w_{i}-\hat{n}_{i}=w_{i}-n_{i}^{*}>0$, where the last inequality follows by the assumption that $w_{i}>n_{i}^{*}$.

Comparing the centralized solution to the decentralized solutions will involve the expectations given in Table2. Let $S_{1}=\sum\left(\gamma_{i}-\tilde{\gamma}\right)$ and $S_{2}=\sum\left(\theta_{i}-\tilde{\theta}\right)$.

Before analyzing the various solutions, we note that from (3) and (6), the trading-sector constraint can be written as

$$
t_{i}=\frac{1}{\lambda}\left(\theta_{i}-\bar{\theta}\right)+\bar{e} .
$$

Member States with a greater than average cost parameter will have greater than average emissions in the trading sector. For the nonstrategic cases, we use (18) to calculate emissions in the trading sector after the equilibrium $e_{i}$ 's have been determined. For the strategic cases, we substitute (18) into the objective function for the Member States before taking the derivative with respect to $e_{i}$. After the equilibrium $e_{i}$ 's have been determined, we use (18) once again to specify emissions in the trading sector.

\section{Centralized Solution}

The solution to the system of equations corresponding to (5) is

$$
e_{i}^{c}=w_{i}+\frac{1}{\mu+\lambda}(\tilde{\theta}-\tilde{\gamma}-\lambda \bar{w})
$$

hence

$$
\bar{e}=\frac{1}{\mu+\lambda}(\tilde{\theta}-\tilde{\gamma}+\mu \bar{w}) .
$$

From the emission-endowment constraint we have

$$
n_{i}^{c}=\frac{1}{\mu+\lambda}(\tilde{\gamma}-\tilde{\theta}+\lambda \bar{w})
$$

From (18) we have

$$
t_{i}^{c}=\frac{1}{\lambda}\left(\theta_{i}-\bar{\theta}\right)+\frac{1}{\mu+\lambda}(\tilde{\theta}-\tilde{\gamma}+\mu \bar{w})
$$




\section{Full-information, Nonstrategic Case}

The first task is to solve the first-order conditions (7). Substituting (6) for $p$ into (7) yields

$$
\gamma_{i}-\mu\left(w_{i}-e_{i}\right)-(\bar{\theta}-\lambda \bar{e})=0 .
$$

Solving the set of $m$ equations for the $m$ unknowns $e_{i}$ gives the solution

$$
e_{i}^{d}=w_{i}-\frac{1}{\mu}\left(\gamma_{i}-\bar{\gamma}\right)+\frac{1}{\mu+\lambda}(\bar{\theta}-\bar{\gamma}-\lambda \bar{w}),
$$

where $\bar{\gamma}$ is the average of the realized $\gamma_{i}$ 's. From $n_{i}+e_{i}=w_{i}$ we have

$$
n_{i}^{d}=\frac{1}{\mu}\left(\gamma_{i}-\bar{\gamma}\right)+\frac{1}{\mu+\lambda}(\bar{\gamma}-\bar{\theta}+\lambda \bar{w}) .
$$

From (18) we have

$$
t_{i}^{d}=\frac{1}{\lambda}\left(\theta_{i}-\bar{\theta}\right)+\frac{1}{\mu+\lambda}(\bar{\theta}-\bar{\gamma}+\mu \bar{w})
$$

The second task is to compare the decentralized solution to the centralized solution. First note that $t_{i}^{d}=K_{i}+\frac{1}{\mu+\lambda}(\bar{\theta}-\bar{\gamma})$ and $t_{i}^{c}=K_{i}+\frac{1}{\mu+\lambda}(\tilde{\theta}-\tilde{\gamma})$, where $K_{i}=\frac{1}{\lambda}\left(\theta_{i}-\bar{\theta}\right)+\frac{\mu}{\mu+\lambda} \bar{w}$. Consider the expected costs in the trading sector for Member State $i$. Let $\Delta_{i}^{t}=\mathcal{E}\left[c_{i}^{t}\left(t_{i}^{d}\right)\right]-$ $\mathcal{E}\left[c_{i}^{t}\left(t_{i}^{c}\right)\right]$. We have

$$
\begin{aligned}
\Delta_{i}^{t}= & \mathcal{E}\left[D_{i}-\theta_{i}\left(K_{i}+\frac{1}{\mu+\lambda}(\bar{\theta}-\bar{\gamma})\right)+(\lambda / 2)\left(K_{i}+\frac{1}{\mu+\lambda}(\bar{\theta}-\bar{\gamma})\right)^{2}\right] \\
& -\mathcal{E}\left[D_{i}-\theta_{i}\left(K_{i}+\frac{1}{\mu+\lambda}(\tilde{\theta}-\tilde{\gamma})\right)+(\lambda / 2)\left(K_{i}+\frac{1}{\mu+\lambda}(\tilde{\theta}-\tilde{\gamma})\right)^{2}\right] .
\end{aligned}
$$

Canceling and collecting terms gives us

$$
\begin{aligned}
= & \frac{1}{\mu+\lambda} \mathcal{E}\left[\theta_{i}((\tilde{\theta}-\tilde{\gamma})-(\bar{\theta}-\bar{\gamma}))\right]+\frac{\lambda}{2(\mu+\lambda)} \mathcal{E}\left[2 K_{i}((\bar{\theta}-\bar{\gamma})-(\tilde{\theta}-\tilde{\gamma}))\right] \\
& +\frac{\lambda}{2(\mu+\lambda)^{2}} \mathcal{E}\left[(\bar{\theta}-\bar{\gamma})^{2}-(\tilde{\theta}-\tilde{\gamma})^{2}\right] \\
= & \frac{1}{\mu+\lambda} \mathcal{E}\left[\left(-\theta_{i}+\lambda K_{i}\right)((\bar{\theta}-\bar{\gamma})-(\tilde{\theta}-\tilde{\gamma}))\right] \\
& +\frac{\lambda}{2(\mu+\lambda)^{2}} \mathcal{E}\left[\bar{\theta}^{2}-2 \bar{\theta} \bar{\gamma}+\bar{\gamma}^{2}-\tilde{\theta}^{2}+2 \tilde{\theta} \tilde{\gamma}-\tilde{\gamma}^{2}\right] \\
= & \frac{1}{\mu+\lambda} \mathcal{E}\left[\left(-\bar{\theta}+\frac{\mu \lambda}{\mu+\lambda} \bar{w}\right)((\bar{\theta}-\bar{\gamma})-(\tilde{\theta}-\tilde{\gamma}))\right]+\frac{\lambda}{2(\mu+\lambda)^{2}}\left(\sigma_{\gamma}^{2} / m+\sigma_{\theta}^{2} / m\right) \\
= & \frac{1}{\mu+\lambda} \mathcal{E}[(-\bar{\theta})((\bar{\theta}-\bar{\gamma})-(\tilde{\theta}-\tilde{\gamma}))]+\frac{\lambda}{2(\mu+\lambda)^{2}}\left(\sigma_{\gamma}^{2} / m+\sigma_{\theta}^{2} / m\right) \\
= & \frac{1}{\mu+\lambda}\left(-\left(\sigma_{\theta}^{2} / m+\tilde{\theta}^{2}\right)+\tilde{\theta}^{2}\right)+\frac{\lambda}{2(\mu+\lambda)^{2}}\left(\sigma_{\gamma}^{2} / m+\sigma_{\theta}^{2} / m\right) \\
= & -\frac{1}{\mu+\lambda}\left(\frac{\sigma_{\theta}^{2}}{m}\right)+\frac{\lambda}{2(\mu+\lambda)^{2}}\left(\frac{\sigma_{\gamma}^{2}}{m}+\frac{\sigma_{\theta}^{2}}{m}\right) .
\end{aligned}
$$


Turning to the non-trading sector, we have $n_{i}^{c}=K+\frac{1}{\mu+\lambda}(\tilde{\gamma}-\tilde{\theta})$ and $n_{i}^{d}=K+\frac{1}{\mu+\lambda}$ $(\bar{\gamma}-\bar{\theta})+\frac{1}{\mu}\left(\gamma_{i}-\bar{\gamma}\right)$, where $K=\frac{\lambda}{\mu+\lambda} \bar{w}$. Let $\Delta_{i}^{n}=\mathcal{E}\left[c_{i}^{n}\left(n_{i}^{d}\right)\right]-\mathcal{E}\left[c_{i}^{n}\left(n_{i}^{c}\right)\right]$. We have

$$
\begin{aligned}
\Delta_{i}^{n}=\mathcal{E} & {\left[A_{i}-\gamma_{i}\left(K+\frac{1}{\mu+\lambda}(\bar{\gamma}-\bar{\theta})+\frac{1}{\mu}\left(\gamma_{i}-\bar{\gamma}\right)\right)\right.} \\
& \left.+\frac{\mu}{2}\left(K+\frac{1}{\mu+\lambda}(\bar{\gamma}-\bar{\theta})+\frac{1}{\mu}\left(\gamma_{i}-\bar{\gamma}\right)\right)^{2}\right] \\
-\mathcal{E} & {\left[A_{i}-\gamma_{i}\left(K+\frac{1}{\mu+\lambda}(\tilde{\gamma}-\tilde{\theta})\right)+\frac{\mu}{2}\left(K+\frac{1}{\mu+\lambda}(\tilde{\gamma}-\tilde{\theta})\right)^{2}\right] . }
\end{aligned}
$$

Canceling terms leaves us with

$$
\begin{aligned}
= & \mathcal{E}\left[-\frac{1}{\mu+\lambda} \gamma_{i}(\bar{\gamma}-\bar{\theta})-\frac{1}{\mu} \gamma_{i}\left(\gamma_{i}-\bar{\gamma}\right)\right] \\
& +\frac{\mu}{2} \mathcal{E}\left[\frac{2 K}{\mu+\lambda}(\bar{\gamma}-\bar{\theta})+\frac{2}{\mu(\mu+\lambda)}(\bar{\gamma}-\bar{\theta})\left(\gamma_{i}-\bar{\gamma}\right)+\frac{2 K}{\mu}\left(\gamma_{i}-\bar{\gamma}\right)\right] \\
& +\frac{\mu}{2} \mathcal{E}\left[K^{2}+\frac{1}{(\mu+\lambda)^{2}}(\bar{\gamma}-\bar{\theta})^{2}+\frac{1}{\mu^{2}}\left(\gamma_{i}-\bar{\gamma}\right)^{2}\right] \\
& -\mathcal{E}\left[\frac{1}{\mu+\lambda} \gamma_{i}(\tilde{\gamma}-\tilde{\theta})\right] \\
& -\frac{\mu}{2} \mathcal{E}\left[K^{2}+\frac{2 K}{\mu+\lambda}(\tilde{\gamma}-\tilde{\theta})+\frac{1}{(\mu+\lambda)^{2}}(\tilde{\gamma}-\tilde{\theta})^{2}\right]
\end{aligned}
$$

Evaluating the expectations yields

$$
\begin{aligned}
= & -\frac{1}{\mu+\lambda}\left(\frac{\sigma_{\gamma}^{2}}{m}+\tilde{\gamma}^{2}-\tilde{\gamma} \tilde{\theta}\right)-\frac{1}{\mu}\left(\sigma_{\gamma}^{2}-\frac{\sigma_{\gamma}^{2}}{m}\right) \\
& +\frac{\mu}{2}\left(\frac{2 K}{\mu+\lambda}(\tilde{\gamma}-\tilde{\theta})+0+0\right) \\
& +\frac{\mu}{2}\left(\frac{1}{(\mu+\lambda)^{2}}\left(\frac{\sigma_{\gamma}^{2}}{m}+\frac{\sigma_{\theta}^{2}}{m}+\tilde{\gamma}^{2}+\tilde{\theta}^{2}+2 \tilde{\gamma} \tilde{\theta}\right)+\frac{1}{\mu^{2}}\left(\sigma_{\gamma}^{2}-\frac{\sigma_{\gamma}^{2}}{m}\right)\right) \\
& +\frac{1}{\mu+\lambda}\left(\tilde{\gamma}^{2}-\tilde{\gamma} \tilde{\theta}\right) \\
& -\frac{\mu}{2}\left(\frac{2 K}{\mu+\lambda}(\tilde{\gamma}-\tilde{\theta})+\frac{1}{(\mu+\lambda)^{2}}(\tilde{\gamma}-\tilde{\theta})^{2}\right)
\end{aligned}
$$

Canceling terms gives

$$
\begin{aligned}
& =-\frac{1}{\mu+\lambda} \frac{\sigma_{\gamma}^{2}}{m}-\frac{1}{\mu}\left(\sigma_{\gamma}^{2}-\frac{\sigma_{\gamma}^{2}}{m}\right)+\frac{1}{2 \mu}\left(\sigma_{\gamma}^{2}-\frac{\sigma_{\gamma}^{2}}{m}\right)+\frac{\mu}{2(\mu+\lambda)^{2}}\left(\frac{\sigma_{\gamma}^{2}}{m}+\frac{\sigma_{\theta}^{2}}{m}\right) \\
& =-\frac{1}{2 \mu} \sigma_{\gamma}^{2}+\left(-\frac{1}{\mu+\lambda}+\frac{1}{\mu}-\frac{1}{2 \mu}+\frac{\mu}{2(\mu+\lambda)^{2}}\right) \frac{\sigma_{\gamma}^{2}}{m}+\frac{\mu}{2(\mu+\lambda)^{2}} \frac{\sigma_{\theta}^{2}}{m} \\
& =-\frac{1}{2 \mu} \sigma_{\gamma}^{2}+\frac{\lambda^{2}}{2 \mu(\mu+\lambda)^{2}}\left(\frac{\sigma_{\gamma}^{2}}{m}\right)+\frac{\mu}{2(\mu+\lambda)^{2}}\left(\frac{\sigma_{\theta}^{2}}{m}\right) .
\end{aligned}
$$


The difference in expected total costs is given by

$$
\begin{aligned}
\mathcal{C}^{d}-\mathcal{C}^{c} & =\sum\left(\mathcal{E}\left[c_{i}^{t}\left(t_{i}^{d}\right)\right]+\mathcal{E}\left[c_{i}^{n}\left(n_{i}^{d}\right)\right]\right)-\sum\left(\mathcal{E}\left[c_{i}^{t}\left(t_{i}^{c}\right)\right]+\mathcal{E}\left[c_{i}^{n}\left(n_{i}^{c}\right)\right]\right) \\
& =\sum\left(\mathcal{E}\left[c_{i}^{t}\left(t_{i}^{d}\right)\right]-\mathcal{E}\left[\left(c_{i}^{t}\left(t_{i}^{c}\right)\right]\right)+\sum\left(\mathcal{E}\left[c_{i}^{n}\left(n_{i}^{d}\right)\right]-\mathcal{E}\left[c_{i}^{n}\left(n_{i}^{c}\right)\right]\right)\right. \\
& =\sum\left(\Delta_{i}^{t}+\Delta_{i}^{n}\right)
\end{aligned}
$$

Substituting (20) and (21) yields

$$
\begin{aligned}
= & \sum\left(-\frac{1}{\mu+\lambda}\left(\frac{\sigma_{\theta}^{2}}{m}\right)+\frac{\lambda}{2(\mu+\lambda)^{2}}\left(\frac{\sigma_{\gamma}^{2}}{m}+\frac{\sigma_{\theta}^{2}}{m}\right)\right) \\
& +\sum\left(-\frac{1}{2 \mu} \sigma_{\gamma}^{2}+\frac{\lambda^{2}}{2 \mu(\mu+\lambda)^{2}}\left(\frac{\sigma_{\gamma}^{2}}{m}\right)+\frac{\mu}{2(\mu+\lambda)^{2}}\left(\frac{\sigma_{\theta}^{2}}{m}\right)\right) \\
= & -\frac{\sigma_{\theta}^{2}}{\mu+\lambda}+\frac{\lambda \sigma_{\gamma}^{2}}{2(\mu+\lambda)^{2}}+\frac{\lambda}{2(\mu+\lambda)^{2}} \sigma_{\theta}^{2}-\frac{m}{2 \mu} \sigma_{\gamma}^{2}+\frac{\lambda^{2} \sigma_{\gamma}^{2}}{2 \mu(\mu+\lambda)^{2}}+\frac{\mu \sigma_{\theta}^{2}}{2(\mu+\lambda)^{2}} \\
= & \frac{\sigma_{\gamma}^{2}}{2 \mu}\left(\frac{\lambda}{\mu+\lambda}-m\right)-\frac{\sigma_{\theta}^{2}}{2(\mu+\lambda)} .
\end{aligned}
$$

\section{Full-information, Strategic Case}

The first-order condition (10) yields a set of $m$ equations for the $m$ unknowns $e_{i}$. Solving this system (and abusing the notation by again referring to the solution as $e_{i}^{d}$ ) gives

$$
\begin{aligned}
e_{i}^{d}= & \frac{\mu}{\mu+\lambda / m}\left(w_{i}-\bar{w}\right)+\frac{1}{m(\mu+\lambda / m)}\left(\theta_{i}-\bar{\theta}\right)-\frac{1}{\mu+\lambda / m}\left(\gamma_{i}-\bar{\gamma}\right) \\
& +\frac{1}{\mu+\lambda}(\bar{\theta}-\bar{\gamma}+\mu \bar{w}) .
\end{aligned}
$$

Notice that the average supply of permits, $\bar{e}$, will be the same as in the full-information, nonstrategic case. It follows that the allocation of emissions in the trading sector will be the same and

$$
\Delta_{i}^{t}=-\frac{1}{\mu+\lambda}\left(\frac{\sigma_{\theta}^{2}}{m}\right)+\frac{\lambda}{2(\mu+\lambda)^{2}}\left(\frac{\sigma_{\gamma}^{2}}{m}+\frac{\sigma_{\theta}^{2}}{m}\right) .
$$

Turning to the non-trading sector, we can write $n_{i}^{d}$ as

$$
n_{i}^{d}=\frac{1}{m b}\left(\lambda\left(w_{i}-\bar{w}\right)+\left(\bar{\theta}-\theta_{i}\right)\right)+\frac{1}{\mu+\lambda}(-\bar{\theta}+\lambda \bar{w})+\frac{1}{\mu+\lambda} \bar{\gamma}+\frac{1}{b}\left(\gamma_{i}-\bar{\gamma}\right),
$$

where $b=\mu+\lambda / m$. So we write $n_{i}^{d}=B_{i}+K+\frac{1}{\mu+\lambda}(\bar{\gamma}-\bar{\theta})+\frac{1}{b}\left(\gamma_{i}-\bar{\gamma}\right)$ and $n_{i}^{c}=$ $K+\frac{1}{\mu+\lambda}(\tilde{\gamma}-\tilde{\theta})$, where $B_{i}=\frac{1}{m b}\left(\lambda\left(w_{i}-\bar{w}\right)+\left(\bar{\theta}-\theta_{i}\right)\right)$. For the decentralized system we have

$$
\begin{aligned}
\mathcal{E}\left[c_{i}^{n}\left(n_{i}^{d}\right)\right]=\mathcal{E} & {\left[A_{i}-\gamma_{i}\left(B_{i}+K+\frac{1}{\mu+\lambda}(\bar{\gamma}-\bar{\theta})+\frac{1}{b}\left(\gamma_{i}-\bar{\gamma}\right)\right)\right.} \\
& \left.+\frac{\mu}{2}\left(B_{i}+K+\frac{1}{\mu+\lambda}(\bar{\gamma}-\bar{\theta})+\frac{1}{b}\left(\gamma_{i}-\bar{\gamma}\right)\right)^{2}\right] .
\end{aligned}
$$


Likewise for the centralized system we have

$$
\mathcal{E}\left[c_{i}^{n}\left(n_{i}^{c}\right)\right]=\mathcal{E}\left[A_{i}-\gamma_{i}\left(K+\frac{1}{\mu+\lambda}(\tilde{\gamma}-\tilde{\theta})\right)+(\mu / 2)\left(K+\frac{1}{\mu+\lambda}(\tilde{\gamma}-\tilde{\theta})\right)^{2}\right] .
$$

We now proceed as before. Expand the terms in (24) and (25), subtract (25) from (24), collect terms, and take the expectation. It follows that

$$
\begin{aligned}
\Delta_{i}^{n}= & -\frac{\lambda}{m b} \tilde{\gamma}\left(w_{i}-\bar{w}\right)-\frac{1}{\mu+\lambda} \frac{\sigma_{\gamma}^{2}}{m}-\frac{1}{b}\left(\sigma_{\gamma}^{2}-\frac{\sigma_{\gamma}^{2}}{m}\right) \\
+ & \frac{\mu}{2}\left(\frac{1}{(m b)^{2}}\left(\lambda^{2}\left(w_{i}-\bar{w}\right)^{2}+\sigma_{\theta}^{2}-\frac{\sigma_{\theta}^{2}}{m}\right)\right. \\
& \left.+\frac{2 K}{m b} \lambda\left(w_{i}-\bar{w}\right)+\frac{2}{m b} \frac{1}{\mu+\lambda}\left(\lambda\left(w_{i}-w\right)(\tilde{\gamma}-\tilde{\theta})\right)\right) \\
+ & \frac{\mu}{2}\left(\frac{1}{(\mu+\lambda)^{2}}\left(\frac{\sigma_{\gamma}^{2}}{m}+\frac{\sigma_{\theta}^{2}}{m}\right)+\frac{1}{b^{2}}\left(\sigma_{\gamma}^{2}-\frac{\sigma_{\gamma}^{2}}{m}\right)\right) .
\end{aligned}
$$

Using (23) and (26), as well as the fact that $\sum\left(w_{i}-\bar{w}\right)=0$, we have

$$
\begin{aligned}
\mathcal{C}^{d}-\mathcal{C}^{c}= & \sum\left(\Delta_{i}^{t}+\Delta_{i}^{n}\right) \\
= & -\frac{1}{\mu+\lambda} \sigma_{\theta}^{2}+\frac{\lambda}{2(\mu+\lambda)^{2}}\left(\sigma_{\gamma}^{2}+\sigma_{\theta}^{2}\right) \\
& -\frac{1}{\mu+\lambda} \sigma_{\gamma}^{2}-\frac{1}{b}\left(m \sigma_{\gamma}^{2}-\sigma_{\gamma}^{2}\right)+\frac{\mu}{2(m b)^{2}}\left(\lambda^{2} \sum\left(w_{i}-\bar{w}\right)^{2}+m \sigma_{\theta}^{2}-\sigma_{\theta}^{2}\right) \\
& +\frac{\mu}{2(\mu+\lambda)^{2}}\left(\sigma_{\gamma}^{2}+\sigma_{\theta}^{2}\right)+\frac{\mu}{2 b^{2}}\left(m \sigma_{\gamma}^{2}-\sigma_{\gamma}^{2}\right) .
\end{aligned}
$$

This can be simplified to

$$
\begin{aligned}
\mathcal{C}^{d}-\mathcal{C}^{c}= & \frac{\mu \lambda^{2}}{2 m b^{2}} \operatorname{var}(w) \\
& +\sigma_{\gamma}^{2}\left(-(m-1) \frac{\mu / 2+\lambda / m}{b^{2}}-\frac{1}{2(\mu+\lambda)}\right) \\
& +\sigma_{\theta}^{2} \frac{1}{2(m b)^{2}(\mu+\lambda)}\left(\mu^{2}\left(m-1-m^{2}\right)-(m+1) \lambda \mu-\lambda^{2}\right) .
\end{aligned}
$$

\section{Private-information, Nonstrategic Case}

This is similar to the full-information, nonstrategic case. Substituting (6) for $p$ into (14) yields

$$
\gamma_{i}-\mu\left(w_{i}-e_{i}\right)-\mathcal{E}\left[\bar{\theta}-\lambda \bar{e} \mid \gamma_{i}\right]=0 .
$$

Solving the resulting set of $m$ equations for the $m$ unknowns $e_{i}$ gives the solution

$$
e_{i}^{d}=w_{i}-\frac{1}{b}\left(\gamma_{i}-\tilde{\gamma}\right)+\frac{1}{\mu+\lambda}(\tilde{\theta}-\tilde{\gamma}-\lambda \bar{w})+\frac{1}{m b}\left(\theta_{i}-\tilde{\theta}\right),
$$


from which it follows that

$$
n_{i}^{d}=\frac{1}{b}\left(\gamma_{i}-\tilde{\gamma}\right)+\frac{1}{\mu+\lambda}(-\tilde{\theta}+\tilde{\gamma}+\lambda \bar{w})-\frac{1}{m b}\left(\theta_{i}-\tilde{\theta}\right)
$$

and

$$
t_{i}^{d}=\frac{1}{\lambda}\left(\theta_{i}-\bar{\theta}\right)-\frac{1}{m b} \sum\left(\gamma_{i}-\tilde{\gamma}\right)+\frac{1}{\mu+\lambda}(\tilde{\theta}-\tilde{\gamma}+\mu \bar{w})+\frac{1}{m^{2} b} \sum\left(\theta_{i}-\tilde{\theta}\right) .
$$

To compare the decentralized solution to the centralized solution we write

$$
n_{i}^{d}=n_{i}^{c}+\frac{1}{b}\left(\gamma_{i}-\tilde{\gamma}\right)-\frac{1}{m b}\left(\theta_{i}-\tilde{\theta}\right)
$$

and

$$
t_{i}^{d}=t_{i}^{c}-\frac{1}{m b} S_{1}+\frac{1}{m^{2} b} S_{2}
$$

Following the usual steps yields

$$
\mathcal{C}^{d}-\mathcal{C}^{c}=-\sigma_{\gamma}^{2} \frac{m}{2 b}-\frac{1}{2 m b} \sigma_{\theta}^{2} .
$$

\section{Private-Information, Strategic Case}

This is similar to the full-information, strategic case. The first-order condition (17) yields a set of $m$ equations for the $m$ unknowns $e_{i}$. Solving this system gives

$$
e_{i}^{d}=\frac{\mu}{\mu+\lambda / m}\left(w_{i}-\bar{w}\right)+\frac{\frac{2}{m}-\frac{1}{m^{2}}}{a}\left(\theta_{i}-\tilde{\theta}\right)-\frac{1}{a}\left(\gamma_{i}-\tilde{\gamma}\right)+\frac{1}{\mu+\lambda}(\tilde{\theta}-\tilde{\gamma}+\mu \bar{w}),
$$

where $a=\mu+(\lambda / m)+(\lambda / m)(1-1 / m)$. We can write $n_{i}^{d}$ as

$$
n_{i}^{d}=\frac{1}{\mu+\lambda}(\tilde{\gamma}-\tilde{\theta}+\lambda \bar{w})+\frac{\lambda}{m b}\left(w_{i}-\bar{w}\right)-\frac{\frac{2}{m}-\frac{1}{m^{2}}}{a}\left(\theta_{i}-\tilde{\theta}\right)+\frac{1}{a}\left(\gamma_{i}-\tilde{\gamma}\right) .
$$

We also have

$$
t_{i}^{d}=\frac{1}{\lambda}\left(\theta_{i}-\bar{\theta}\right)-\frac{1}{m a} \sum\left(\gamma_{i}-\tilde{\gamma}\right)+\frac{1}{\mu+\lambda}(\tilde{\theta}-\tilde{\gamma}+\mu \bar{w})+\frac{\frac{2}{m}-\frac{1}{m^{2}}}{m a} \sum\left(\theta_{i}-\tilde{\theta}\right) .
$$

To compare the decentralized solution to the centralized solution we write

$$
n_{i}^{d}=n_{i}^{c}+\frac{\lambda}{m b}\left(w_{i}-\bar{w}\right)+\frac{1}{a}\left(\gamma_{i}-\tilde{\gamma}\right)-\frac{\frac{2}{m}-\frac{1}{m^{2}}}{a}\left(\theta_{i}-\tilde{\theta}\right)
$$

and

$$
t_{i}^{d}=t_{i}^{c}-\frac{1}{m a} S_{1}+\frac{\frac{2}{m}-\frac{1}{m^{2}}}{m a} S_{2} .
$$

Following the usual steps yields

$\mathcal{C}^{d}-\mathcal{C}^{c}=\frac{\mu \lambda^{2}}{2 m b^{2}} \operatorname{var}(w)-\sigma_{\gamma}^{2} m^{3} \frac{\left((3 m-2) \lambda+m^{2} \mu\right)}{2\left(m^{2} a\right)^{2}}-\sigma_{\theta}^{2}(2 m-1) \frac{((2 m-1) \lambda+m \mu)}{2\left(m^{2} a\right)^{2}}$. 


\section{References}

Bernheim B, Whinston M (1986) Menu auctions, resource allocation, and economic influence. Q J Econ 101:1-31

Böhringer C, Hoffman T, Lange A, Löschel A, Moslener U (2005) Assessing emission regulation in Europe: an interactive simulation approach. Energy J 26:1-22

Commission of the European Communities (2008) Proposal for a directive of the European Parliament and of the council amending directive 2003/87/EC so as to improve and extend the greenhouse gas emission allowance trading system of the Community. Brussels 23 January 2008

Convery F, Redmond L (2007) Market and price development in the European Union emissions trading scheme. Rev Environ Econ Policy 1:88-111

Hahn R (1984) Market power and transferable property rights. Q J Econ 99:753-765

Kruger J, Oates W, Pizer W (2007) Decentralization in the EU emissions trading scheme and lessons for global policy. Rev Environ Econ Policy 1:112-133

Oates W (1999) An essay on fiscal federalism. J Econ Lit 37:1120-1149

Vives X (2002) Private information, strategic behavior, and efficiency in Cournot markets. Rand J Econ $33: 361-376$ 